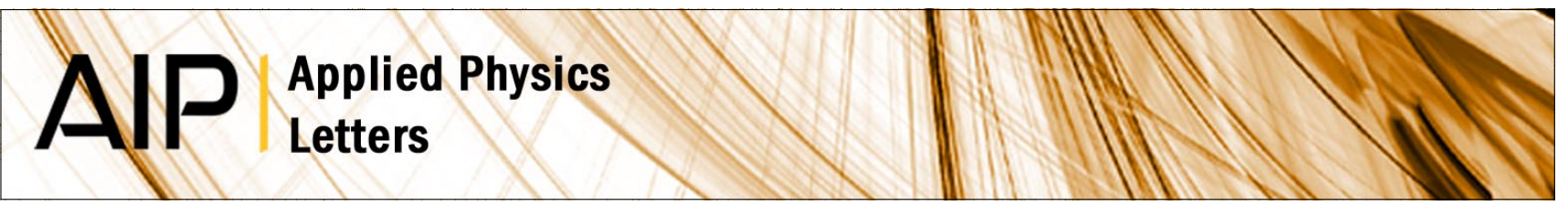

\title{
Complementary chiral metamaterials with giant optical activity and negative refractive index
}

Zhaofeng Li, Kamil Boratay Alici, Evrim Colak, and Ekmel Ozbay

Citation: Appl. Phys. Lett. 98, 161907 (2011); doi: 10.1063/1.3574909

View online: http://dx.doi.org/10.1063/1.3574909

View Table of Contents: http://apl.aip.org/resource/1/APPLAB/v98/i16

Published by the American Institute of Physics.

\section{Related Articles}

Electro-optic chirality control in MgO:PPLN

J. Appl. Phys. 112, 073103 (2012)

Understanding of nonlinear optical properties of CS2 from a microscopic viewpoint

J. Chem. Phys. 137, 084315 (2012)

Nuclear spin optical rotation and Faraday effect in gaseous and liquid water

J. Chem. Phys. 136, 184502 (2012)

Preventing optical deactivation of nanocluster Si sensitized Er using nanometer-thin SiNx/SiO2:Er heterolayer thin film

J. Appl. Phys. 108, 073101 (2010)

Colossal optical activity of split-ring resonator arrays for millimeter waves

Appl. Phys. Lett. 97, 081116 (2010)

\section{Additional information on Appl. Phys. Lett.}

Journal Homepage: http://apl.aip.org/

Journal Information: http://apl.aip.org/about/about_the_journal

Top downloads: http://apl.aip.org/features/most_downloaded

Information for Authors: http://apl.aip.org/authors

\section{ADVERTISEMENT}

\section{AIP Applied Physics Letters}

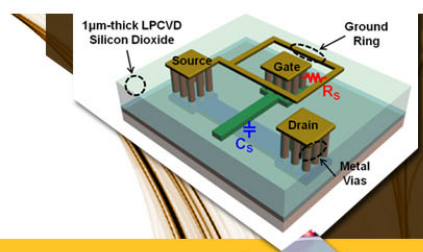

SURFACES AND INTERFACES

Focusing on physical, chemical, biological, structural, optical, magnetic and electrical properties of surfaces and interfaces, and more..

\section{EXPLORE WHAT'S NEW IN APL}

SUBMIT YOUR PAPER NOW!
ENERCY CONVERSION AND STORACE

Focusing on all aspects of static and dynamic energy conversion, energy storage, photovoltaics, solar fuels, batteries, capacitors, thermoelectrics. and more... 


\title{
Complementary chiral metamaterials with giant optical activity and negative refractive index
}

\author{
Zhaofeng $\mathrm{Li}^{1}{ }^{1, \mathrm{a})}$ Kamil Boratay Alici, ${ }^{1}$ Evrim Colak, ${ }^{1}$ and Ekmel Ozbay ${ }^{1,2}$ \\ ${ }^{1}$ Department of Physics, Nanotechnology Research Center, Bilkent University, Bilkent, 06800 Ankara, \\ Turkey \\ ${ }^{2}$ Department of Electrical and Electronics Engineering, Bilkent University, Bilkent, 06800 Ankara, Turkey
}

(Received 1 February 2011; accepted 13 March 2011; published online 20 April 2011)

\begin{abstract}
A complementary bilayer cross-wire chiral metamaterial is proposed and studied experimentally and numerically. It exhibits giant optical activity and a small circular dichroism. The retrieval results reveal that a negative refractive index is realized for right circularly polarized waves due to the strong chirality. Our numerical results show that the mechanism of the chiral behavior at the resonance of lower frequency can be interpreted as the coupling effects between two sets of mutually twisted virtual magnetic dipoles, while the resonance of higher frequency shows complicated nonlocal features. (C) 2011 American Institute of Physics. [doi:10.1063/1.3574909]
\end{abstract}

Recently, chiral metamaterials (CMMs) have attracted much attention due to their exotic properties, e.g., giant optical activity, circular dichroism, and negative refraction. ${ }^{1-13}$ A CMM lacks any mirror symmetry so that the crosscoupling between the electric and magnetic fields exists at the resonance. The degeneracy of the two circularly polarized waves is thereby broken, i.e., the refractive indices of right circularly polarized $(\mathrm{RCP},+)$ waves and left circularly polarized $(\mathrm{LCP},-)$ waves have different values. The strength of the cross-coupling effect can be described by a chirality parameter $\kappa$, so that the constitutive relations of a chiral medium is given by

$$
\left(\begin{array}{l}
D \\
B
\end{array}\right)=\left(\begin{array}{cc}
\varepsilon_{0} \varepsilon & -i \kappa / c \\
i \kappa / c & \mu_{0} \mu
\end{array}\right)\left(\begin{array}{l}
E \\
H
\end{array}\right)
$$

where $\varepsilon_{0}$ and $\mu_{0}$ are the permittivity and permeability of vacuum. $\varepsilon$ and $\mu$ are the relative permittivity and permeability of the chiral medium. $c$ is the speed of light in vacuum. Assuming a time dependence of $e^{-i \omega t}$, the RCP $(+)$ wave and LCP $(-)$ wave are defined as $E^{ \pm}=(1 / 2) E_{0}(\hat{x} \mp i \hat{y})$ when the waves are propagating in the positive $z$ direction. ${ }^{14}$ The refractive indices for RCP and LCP waves can be expressed as $n_{ \pm}=n \pm \kappa,{ }^{15}$ where $n=\sqrt{\varepsilon \mu}$. When $\kappa$ is large enough, either $n_{+}$or $n_{-}$becomes negative. At the same time, both RCP and LCP waves have the same impedance of $z / z_{0}=\sqrt{\mu / \varepsilon}$, where $z_{0}$ is the impedance of the vacuum.

Now, consider two structures as shown in Figs. 1(a) and 1(b), in which one structure is a short thin metal wire, and the other is its complement, i.e., a slot cut in an infinite planar metal sheet. According to Babinet's principle, if the metal wire is normally illuminated from $z<0$ by an incident field $\left(E^{0}, B^{0}\right)$ and its complementary screen is illuminated by a complementary incident field $\left(E_{c}{ }^{0}=-c B^{0}, B_{c}{ }^{0}=E^{0} / c\right)$, then the pattern of the field scattered by the metal wire is the same as that which is scattered by its complement except that the polarization of the fields will be opposite for the two systems. Since the field scattered by the wire can be approximated as the radiation field by an electric dipole when the higher order multipolar fields are negligible, the field scat-

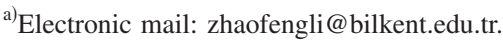

tered by its complement can then be thought of as the radiation field produced by a virtual magnetic dipole. The electric (magnetic) dipoles on the $z<0$ and $z>0$ sides are symmetric (antisymmetric) in order to fulfill the boundary conditions, ${ }^{14,16}$ as shown in the insets of Figs. 1(a) and 1(b). Babinet's principle has been applied to the design of metasurfaces and single-layered metamaterials. ${ }^{16-18}$ However, it is rarely seen that this principle is used to construct multilayered metamaterials. In this letter, we propose a design of a complementary CMM (CCMM) based on the complementary structure of the bilayer cross-wire CMM reported before. $^{10,12}$

Figure 1(c) shows the schematic of one periodic unit cell of the CCMMs which consists of double layered metal (copper) plates patterned on opposite sides of an FR-4 board. Two cross-wires that are mutually twisted by $30^{\circ}$ are cut out from the two metal plates. The relative dielectric constant of

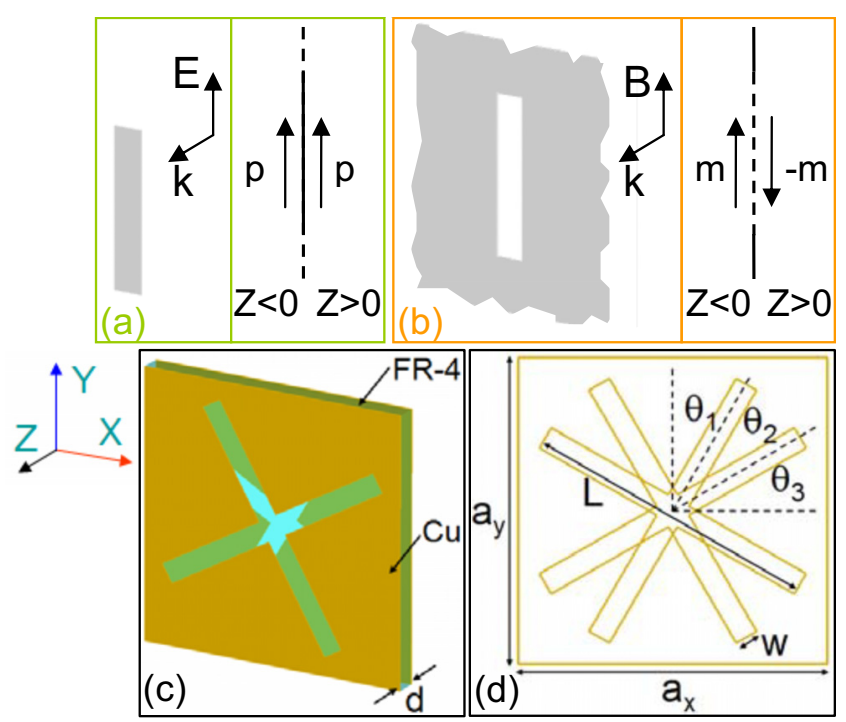

FIG. 1. (Color online) Illustration of the scattering behavior of a short thin metal wire (a) and its complimentary structure (b) when they are illuminated by an external field from $z<0$ according to Babinet's principle. (c) Schematic of a unit cell of the complementary cross-wire pair metamaterial. (d) The structure viewed from front. The parameters are given by $a_{x}=a_{y}$ $=21 \mathrm{~mm}, d=1.6 \mathrm{~mm}, w=1.6 \mathrm{~mm}, L=20 \mathrm{~mm}$, and $\theta_{1}=\theta_{2}=\theta_{3}=30^{\circ}$. The copper has a thickness of $0.03 \mathrm{~mm}$. 


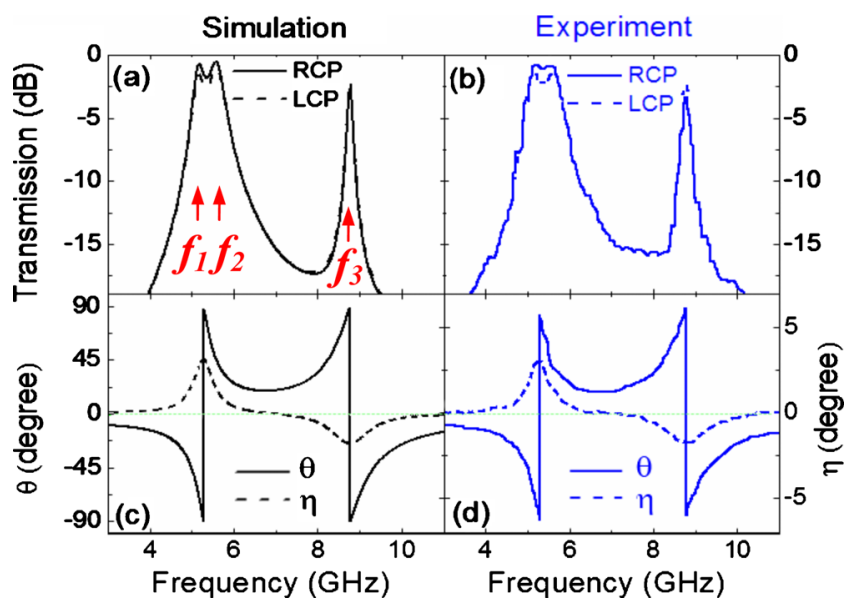

FIG. 2. (Color online) Simulation and experimental results for the CCMM. (a) and (b) are the transmission spectra for RCP and LCP waves. $f_{1}$ $=5.17 \mathrm{GHz}, f_{2}=5.58 \mathrm{GHz}$, and $f_{3}=8.77 \mathrm{GHz}$ indicate three transmission peaks. (c) and (d) are the rotation angle $\theta$ and the ellipticity angle $\eta$ of the transmitted wave.

the FR-4 board is 4.0 with a dielectric loss tangent of 0.025 . The dimensions of the unit cell are shown in Fig. 1(d) and the caption. To study this chiral structure, we conducted numerical simulations and experiments. The simulation works were carried out by using CST microwave studio (Computer Simulation Technology GmbH, Germany), wherein the finite integration technique was applied. The periodic boundary conditions were applied to the $x$ and $y$ directions, and the absorbing boundary conditions were applied to the $z$ direction. In the experiment, we fabricated the chiral structures with a dimension of 18 by 18 unit cells. The transmission coefficient was measured by an HP-8510C network analyzer with two standard horn antennas. A linearly polarized electromagnetic (EM) wave ( $E$ field in the $x$ direction) is incident on the chiral structure. On the other side of the structure, we measured the transmitted field in the $x$ and $y$ polarizations $\left(T_{x x}\right.$ and $\left.T_{y x}\right)$. Due to the fourfold rotational symmetry, circular polarization conversion is absent. The transmission of circularly polarized waves can be converted from the linear transmission coefficients $T_{x x}$ and $T_{y x},{ }^{10} T^{ \pm}=T_{x x} \pm i T_{y x}$. For the transmitted EM wave, the polarization azimuth rotation angle $\theta$ is calculated as $\theta=\left[\arg \left(T^{+}\right)-\arg \left(T^{-}\right)\right] / 2$, and the ellipticity is defined as $\eta=\arctan \left[\left(\left|T^{+}\right|-\left|T^{-}\right|\right) /\left(\left|T^{+}\right|+\left|T^{-}\right|\right)\right],{ }^{12}$ which also measures the circular dichroism.

Figures 2(a) and 2(b) show the simulated and measured transmission spectra. The transmissions of RCP and LCP waves are almost the same except for a little difference (1-2 $\mathrm{dB})$ around the three transmission peaks, i.e., $f_{1}=5.17 \mathrm{GHz}$, $f_{2}=5.58 \mathrm{GHz}$, and $f_{3}=8.77 \mathrm{GHz}$, respectively. Figures $2(\mathrm{c})$ and 2(d) show the results of $\theta$ and $\eta$ of the transmitted wave. It can be clearly seen that the ellipticity $\eta$ is less than $3^{\circ}$ within the whole frequency range. This is almost one order of magnitude smaller than that reported earlier for the fourU-SRRs CMMs (Ref. 13) which used the same lossy FR-4 board but had $\eta$ larger than $20^{\circ}$ at resonances. At the frequency $f=6.9 \mathrm{GHz}, \eta=0$ where it corresponds to a pure optical activity effect, i.e., for the linearly polarized incident wave, the transmission wave is still linearly polarized but with a rotated angle $\theta$. The azimuth rotation angle $\theta$ is approximately $20^{\circ}$ at $6.9 \mathrm{GHz}$, which is more than $500^{\circ}$ per wavelength.

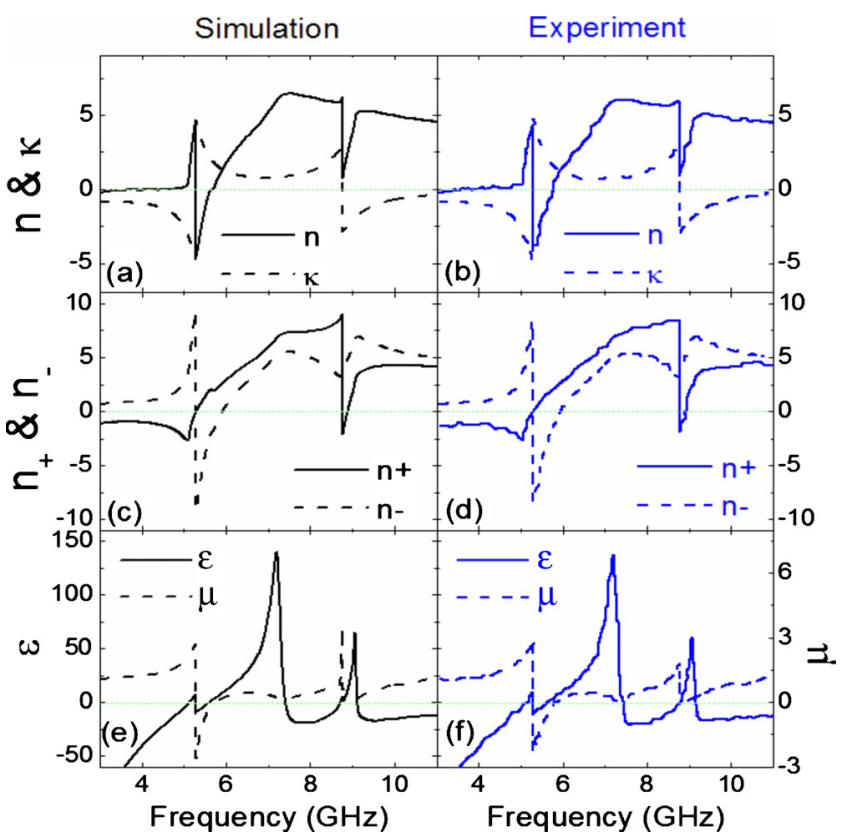

FIG. 3. (Color online) The retrieved effective parameters of the CCMM based on the simulation (left) and experimental (right) data. (a) and (b) show the real parts of the $n$ and $\kappa$. (c) and (d) show the real parts of the $n_{+}$and $n_{-}$. (e) and (f) show the real parts of the $\varepsilon$ and $\mu$.

Figure 3 shows the retrieved effective parameters based on the simulation and experimental data of the transmission and reflection for one layer of the CCMMs. ${ }^{19}$ Here, the effective thickness of the CMM is assumed to be $3.0 \mathrm{~mm}$ along the wave propagation direction. On the $\kappa$ curves shown in Figs. 3(a) and 3(b), there are two resonances related to the chirality. The lower frequency resonance happens at $f$ $=5.28 \mathrm{GHz}$, and the upper one happens at $f=8.77 \mathrm{GHz}$. Below $f=5.28 \mathrm{GHz}, n$ is positive while $\kappa$ is negative. Above this frequency $n$ is negative and $\kappa$ is positive. For $f$ $=8.77 \mathrm{GHz}$, only $\kappa$ changes its sign while $n$ remains positive on both sides. Comparing Figs. 3(a) and 3(b) with Figs. 3(c) and 3(d), due to the relation of $n_{ \pm}=n \pm \kappa$, the strong chirality $\kappa$ has pushed the refractive index of the RCP wave from positive to negative values below $f=5.28 \mathrm{GHz}$ and above $f=8.77 \mathrm{GHz}$. At the same time, above $f=5.28 \mathrm{GHz}$, the originally negative index band of the LCP wave becomes wider. For the RCP wave, the figure of merit $\left[-\operatorname{Re}\left(n_{+}\right) / \operatorname{Im}\left(n_{+}\right)\right]$is around $5.2(4.5)$ at $5.16(8.80) \mathrm{GHz}$ in the negative index band. For the LCP wave, the figure of merit is larger than 15 at $5.3 \mathrm{GHz}$ in the negative index band. These values are higher than that reported earlier. ${ }^{13}$ In Figs. $3(\mathrm{e})$ and 3(f), we show the retrieved real parts of the $\varepsilon$ and $\mu$. Obviously, due to the presence of the continuous metal plate, there exists a plasma frequency $f=5.10 \mathrm{GHz}$ below which the $\operatorname{Re}(\varepsilon)$ is always negative. Corresponding to the two resonance frequencies $(5.28$ and $8.77 \mathrm{GHz})$ of the $\kappa$ curve, there are two obvious resonances on the curve of $\operatorname{Re}(\mu)$, and an obvious resonance and a tiny resonance on the curve of $\operatorname{Re}(\varepsilon)$. Besides the above two resonances, there are two huge resonances on the curve of $\operatorname{Re}(\varepsilon)$ located at $7.2 \mathrm{GHz}$ and 9.0 $\mathrm{GHz}$, respectively. However, they do not affect the chiral behavior and will not be discussed here. It is noteworthy that in the frequency ranges $5.10-5.28 \mathrm{GHz}$ and $8.77-8.90 \mathrm{GHz}$, both the $\operatorname{Re}(\mu)$ and $\operatorname{Re}(\varepsilon)$ are positive and will not result in a negative index in traditional metamaterials. Therefore, the 


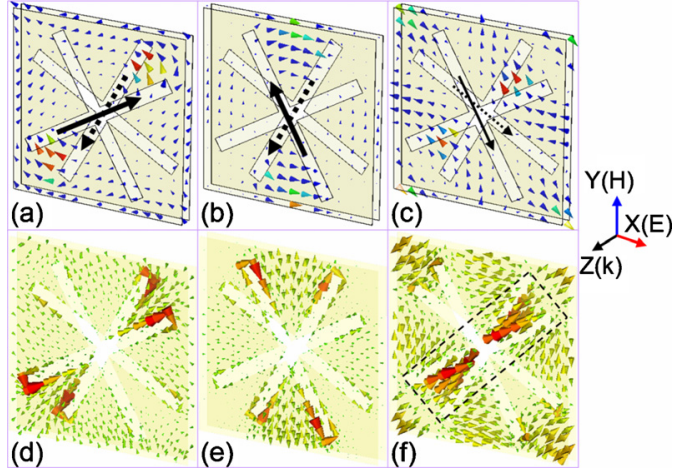

FIG. 4. (Color online) The scattered $H$ field distributions on the middle plane between the two metal plates and the current modes when driven by the incident field with $E$ in the $x$ direction at (a) and (d) $5.17 \mathrm{GHz}$, (b) and (e) $5.58 \mathrm{GHz}$, and (c) and (f) $8.77 \mathrm{GHz}$. The long solid (dashed) arrows represent the front (back) virtual magnetic dipoles.

negative index of RCP wave is actually attributed to the relatively small $n$ and large chirality $\kappa$.

For further understanding the mechanism of the chiral behaviors, we now study the distribution of the $H$ fields on the middle plane between the two metal plates and the surface current modes. Figure 4 shows the simulated $H$ field distributions and the current modes at the frequencies of three transmission peaks, e.g., Fig. 4(a) and 4(d) at $f_{1}$ $=5.17 \mathrm{GHz}$, Fig. 4(b) and 4(e) at $f_{2}=5.58 \mathrm{GHz}$, and Fig. $4(\mathrm{c})$ and $4(\mathrm{f})$ at $f_{3}=8.77 \mathrm{GHz}$, respectively. For all three cases, the incident waves have the $E$ field polarized in the $x$ direction. From Fig. 4(a) and 4(d), one sees that both the distribution of the $H$ field and the current mode resemble the coupling of a pair of antisymmetrically arranged magnetic dipoles. The two virtual magnetic dipoles are depicted in Fig. 4(a), with the thick solid (dashed) arrow representing the front (back) virtual magnetic dipole. The angle is $30^{\circ}$ between the two dipoles. For the case of Figs. 4(b) and 4(e), the distribution of $H$ field and current mode also resemble the coupling of a pair of antisymmetrically arranged magnetic dipoles. The only difference is the angle, which is $60^{\circ}$ in this case. However, Figs. 4(c) and 4(f) are complicated. Both the $H$ field and current mode are not well confined to the slots. Only the $H$ field on the corners partially resembles the coupling of two symmetric magnetic dipoles [thin arrows in Fig. 4(c)], while the main part of the $H$ field in the center region is attributed to the antisymmetric currents as indicated with dashed box in Fig. 4(f). These antisymmetric currents function as coupled electric dipoles instead of magnetic dipoles. In all three cases, the net values of the induced $H$ fields are nonzero in the $x$ direction. The causality between $E$ and $H$ fields is consistent with Eq. (1). Furthermore, we also investigated numerically the effect of thickness of the middle layer $(d)$ on the transmission and optical activity. When $d$ increases, the coupling between the two metal plates be- comes weaker so that the optical activity becomes lower and the frequency gap between the transmission peaks $f_{1}\left(f_{2}\right)$ and $f_{3}$ decreases.

In summary, we have demonstrated the chiral properties of a CCMM. Like its counterpart (the cross-wire CMM), the CCMM also shows giant optical activity, and due to the strong chirality, the frequency bands of negative index are obtained for RCP waves. The mechanism of the chiral behavior at the resonance of lower frequency can be interpreted as the coupling effects between two sets of mutually twisted virtual magnetic dipoles, but the resonance of higher frequency shows complicated nonlocal features which cannot be interpreted simply with a model of virtual magnetic dipoles.

This work is supported by the European Union under the projects PHOME, ECONAM, and N4E, and TUBITAK under Project Nos. 109E301, 107A004, and 107A012, and DPT under the project DPT-HAMIT. One of the authors (E.O.) also acknowledges partial support from the Turkish Academy of Sciences.

${ }^{1}$ J. B. Pendry, Science 306, 1353 (2004).

${ }^{2}$ S. Tretyakov, I. Nefedov, A. Sihvola, S. Maslovski, and C. Simovski, J. Electromagn. Waves Appl. 17, 695 (2003).

${ }^{3}$ A. V. Rogacheva, V. A. Fedotov, A. S. Schwanecke, and N. I. Zheludev, Phys. Rev. Lett. 97, 177401 (2006).

${ }^{4}$ H. Liu, D. A. Genov, D. M. Wu, Y. M. Liu, Z. W. Liu, C. Sun, S. N. Zhu, and X. Zhang, Phys. Rev. B 76, 073101 (2007)

${ }^{5}$ T. Q. Li, H. Liu, T. Li, S. M. Wang, F. M. Wang, R. X. Wu, P. Chen, S. N. Zhu, and X. Zhang, Appl. Phys. Lett. 92, 131111 (2008).

${ }^{6}$ N. Liu, H. Liu, S. Zhu, and H. Giessen, Nat. Photonics 3, 157 (2009).

${ }^{7}$ B. Wang, J. Zhou, T. Koschny, and C. M. Soukoulis, Appl. Phys. Lett. 94, 151112 (2009).

${ }^{8}$ S. Zhang, Y.-S. Park, J. Li, X. Lu, W. Zhang, and X. Zhang, Phys. Rev. Lett. 102, 023901 (2009).

${ }^{9}$ E. Plum, J. Zhou, J. Dong, V. A. Fedotov, T. Koschny, C. M. Soukoulis, and N. I. Zheludev, Phys. Rev. B 79, 035407 (2009).

${ }^{10}$ J. Zhou, J. Dong, B. Wang, T. Koschny, M. Kafesaki, and C. M. Soukoulis, Phys. Rev. B 79, 121104(R) (2009).

${ }^{11}$ M. Decker, M. Ruther, C. E. Kriegler, J. Zhou, C. M. Soukoulis, S. Linden, and M. Wegener, Opt. Lett. 34, 2501 (2009).

${ }^{12}$ Z. Li, H. Caglayan, E. Colak, J. Zhou, C. M. Soukoulis, and E. Ozbay, Opt. Express 18, 5375 (2010).

${ }^{13}$ Z. Li, R. Zhao, T. Koschny, M. Kafesaki, K. B. Alici, E. Colak, H. Caglayan, E. Ozbay, and C. M. Soukoulis, Appl. Phys. Lett. 97, 081901 (2010).

${ }^{14}$ J. D. Jackson, Classical Electrodynamics, 3rd ed. (Wiley, New York, 1998).

${ }^{15}$ J. A. Kong, Electromagnetic Wave Theory (EMW, Cambridge, MA, 2008).

${ }^{16}$ F. Falcone, T. Lopetegi, M. A. G. Laso, J. D. Baena, J. Bonache, M. Beruete, R. Marques, F. Martin, and M. Sorolla, Phys. Rev. Lett. 93, 197401 (2004).

${ }^{17}$ T. H. Hand, J. Gollub, S. Sajuyigbe, D. R. Smith, and S. A. Cummer, Appl. Phys. Lett. 93, 212504 (2008).

${ }^{18}$ N. Liu, S. Kaiser, and H. Giessen, Adv. Mater. (Weinheim, Ger.) 20, 4521 (2008).

${ }^{19}$ R. Zhao, Th. Koschny, E. N. Economou, and C. M. Soukoulis, Phys. Rev. B 81, 235126 (2010). 